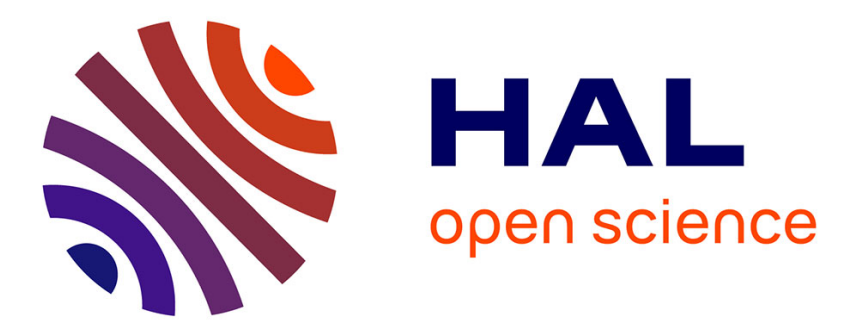

\title{
Optimisation d'un transformateur a courant continu pour le réglage fin de l'excitation d'une lentille magnétique supraconductrice "en boucle fermée"
}

D. Genotel, G. Balossier

\section{- To cite this version:}

D. Genotel, G. Balossier. Optimisation d'un transformateur a courant continu pour le réglage fin de l'excitation d'une lentille magnétique supraconductrice "en boucle fermée". Revue de Physique Appliquée, 1975, 10 (6), pp.443-451. 10.1051/rphysap:01975001006044300 . jpa-00243944

HAL Id: jpa-00243944

https://hal.science/jpa-00243944

Submitted on 1 Jan 1975

HAL is a multi-disciplinary open access archive for the deposit and dissemination of scientific research documents, whether they are published or not. The documents may come from teaching and research institutions in France or abroad, or from public or private research centers.
L'archive ouverte pluridisciplinaire $\mathbf{H A L}$, est destinée au dépôt et à la diffusion de documents scientifiques de niveau recherche, publiés ou non, émanant des établissements d'enseignement et de recherche français ou étrangers, des laboratoires publics ou privés. 


\author{
Classification \\ Physics Abstracts \\ 0.691

\section{OPTIMISATION D'UN TRANSFORMATEUR A COURANT CONTINU POUR LE RÉGLAGE FIN DE L'EXCITATION D'UNE LENTILLE MAGNÉTIQUE SUPRACONDUCTRICE «EN BOUCLE FERMÉE »}

\author{
D. GENOTEL et G. BALOSSIER \\ Laboratoire de Microscopie Electronique (ERA No 376) \\ Université de Reims, U. E. R. Sciences, BP no 347, 51062 Reims Cedex, France
}

(Reçu le 16 mai 1975, accepté le 16 juin 1975)

\begin{abstract}
Résumé. - Pour créer des petites variations de courant électrique dans des lentilles magnétiques supraconductrices fonctionnant en boucle fermée le plus simple est d'utiliser un transformateur à courant continu. Le calcul et l'optimisation des diverses solutions possibles montrent que les transformateurs comportant un circuit magnétique avec entrefer sont les plus intéressants tant du point de vue du champ magnétique parasite créé qui est très faible, que de leur réponse qui est linéaire ou de leurs dimensions qui sont très réduites. Quelques transformateurs optimisés sont présentés et leur performances calculées sont vérifiées expérimentalement.

Abstract. - The most simple method of creating small changes of electric current in superconducting magnetic lenses in the persistent mode is to use direct-current transformers. Iron-clad transformers with an air gap in the core are the most interesting ones because of their weak magnetic stray-field, linear response and small volume. Some optimised transformers are presented and their calculated performances are confirmed experimentally.
\end{abstract}

1. Introduction. - L'utilisation des matériaux supraconducteurs pour la réalisation des enroulements excitateurs dans des lentilles magnétiques présente un certain nombre d'avantages notamment en Microscopie Electronique [1, 2, 3, 4]. Aucune puissance électrique n'est dissipée dans ces enroulements et grâce aux densités de courant très élevées admises par les supraconducteurs, on a des lentilles qui ont de meilleures caractéristiques optiques tout en ayant des dimensions plus petites. Ces lentilles ont d'autre part une stabilité en courant parfaite quand elles fonctionnent en boucle fermée, l'enroulement supraconducteur étant alors fermé sur lui-même par une soudure supraconductrice (Fig. 1).

Pour alimenter ces lentilles en boucle fermée, on utilise un interrupteur de supraconduction placé entre les deux points d'alimentation : cet interrupteur, permet l'établissement et le piégeage d'un courant électrique dans l'enroulement fermé de la lentille (Fig. 2)'.

Ei Microscopie Electronique pour effectuer, la mise au point ou pour réaliser une série foçlé au voisinage de cette mise au pooint, on doit pouvoir

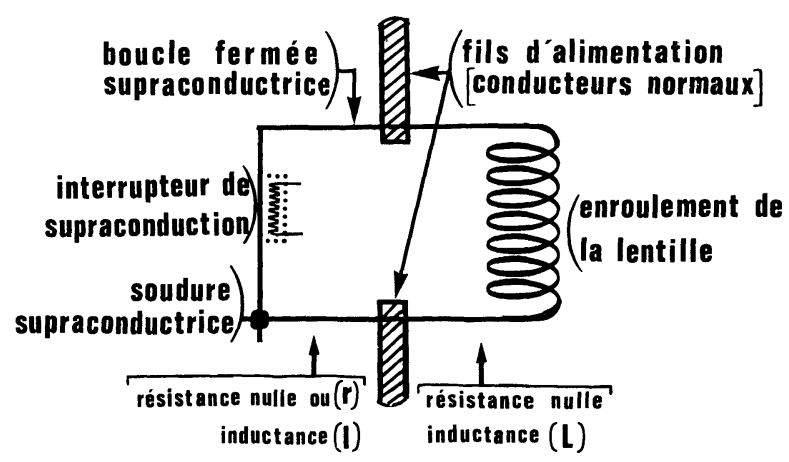

Fig. 1. - Schéma d'un enroulement supraconducteur en boucle fermée.

commander avec précision des variations très petites du courant dans la lentille-objectif. Pour une variation $\Delta I$ de ce courant la défocalisation correspondante $\Delta z$ est donnée par la formule $\Delta z=\alpha_{\circ} f_{0} . \Delta I / I$ où $f_{0}$ est la distance focale-objectif de la lentille et $\alpha$ un coefficient qui dépend de son point de fonctionnement [5] : dans notre microsope [4] nous avons par exemple 


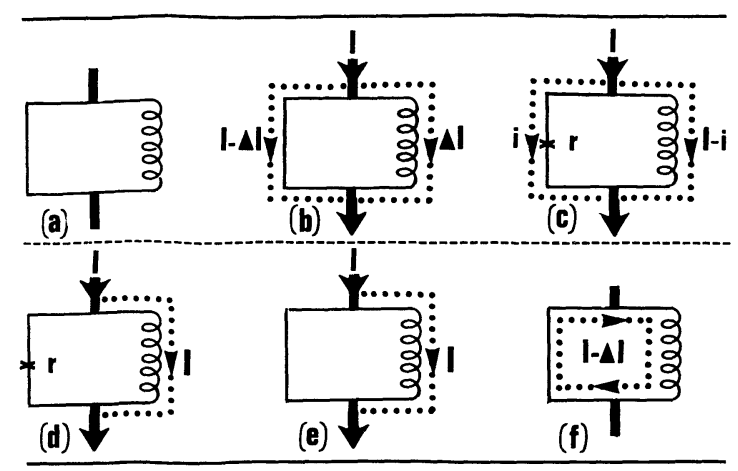

Fig. 2. - Schémas illustrant l'alimentation en courant d'un enroulement en boucle fermée. (a) et $(b):$ la boucle est fermée et le courant piégé est nul. $(c)$ et $(d):$ la boucle est ouverte et le courant extérieur $I$ s'établit progressivement dans l'enroulement. $(e)$ et $(f)$ : la boucle est refermée et un courant y est piégé. Son intensité $I$ devient $I-\Delta I$ quand on coupe l'alimentation extérieure.

$f_{0}=2$ mm et $\alpha \simeq 1,25$ ce qui donne $\Delta I / I=4 \times 10^{-4} . \Delta z$ si $\Delta z$ ést exprimé en microns.

Habituellement avec une profondeur de foyer de quelques $0,1 \mu \mathrm{m}$ on doit pouvoir effectuer des variations relatives de courant $\Delta I / I$ aussi petites que $10^{-5}$. On peut même aller jusqu'à $10^{-6}$ si on fait de la très haute résolution où le contraste de phase permet d'envisager l'effet de défocalisations de l'ordre de $100 \AA$. On ne peut considérer de toute façon que des variations relatives d'un ordre de grandeur supérieur à celui de l'instabilité $\Delta V / V$ du générateur haute tension du microscope.

Nous nous intéresserons ici au problème de la commande de ces petites variations de l'intensité du courant dans une lentille supraconductrice en boucle fermée. Nous considérerons principalement la commande indirecte par transformateur et nous étudierons les différents types de transformateurs pouvant être utilisés. Nous calculerons ces transformateurs en optimisant leurs dimensions : ces transformateurs doivent en effet être placés dans le cryostat à hélium liquide de la lentille supraconductrice et il est de ce fait important qu'ils aient un très faible volume, tant du point de vue de leur placement que de la masse à refroidir introduite dans le cryostat.

2. Commande directe de petites variations de courant. - On peut dans un premier stade envisager de procéder comme pour les variations importantes en ouvrant la boucle supraconductrice avec l'interrupteur et en commandant le courant avec l'alimentation extérieure. Cette méthode nécessite l'emploi d'une alimentation très stable et elle introduit par ailleurs une difficulté qui tient au fait que le courant piégé dans une lentille n'est jamais rigoureusement identique au courant commandé par l'alimentation et ceci pour deux raisons :

- D'une part, le courant ne s'établit que progressivement dans l'enroulement de la lentille avec une constante de temps $\tau$ qui pour nos lentilles est de plusieurs secondes (Fig. $2 c, d$ ). Le courant piégé à un instant donné n'est donc pas identique au courant extérieur au même instant et si on réouvre ensuite la boucle supraconductrice sans modifier l'alimentation le courant continue alors de s'établir. Si on désire contrôler de faibles variations de courant il faut donc que cette variation résiduelle qui intervient à la réouverture de la boucle soit négligeable : cela suppose une temporisation importante avant chaque piégeage de courant. Si on désire par exemple effectuer des variations $\Delta I / I$ de l'ordre de $10^{-5}$, on devra alors avoir précédemment laissé le courant s'établir à $10^{-6}$ ou $10^{-7}$ près ce qui suppose une temporisation d'environ $15 . \tau$ soit plus d'une minute pour nos lentilles.

- D'autre part, le courant piégé dans l'enroulement de la lentille varie systématiquement quand on supprime le courant dans le circuit extérieur d'alimentation. Supposons qu'on ait suffisamment attendu et que le courant $I$ soit entièrement établi et piégé dans la lentille (Fig. 2e) : si on supprime alors le courant d'alimentation (Fig. $2 f$ ) la conservation du flux dans la boucle supraconductrice entraîne une variation de courant $\Delta I$ donnée par $\Delta I / I=l / L, l$ et $L$ étant les coefficients d'inductance des deux branches en parallèle entre les points d'alimentation. Le coefficient d'inductance de la branche qui porte l'interrupteur dépend essentiellement de la conception de l'interrupteur. Pour nos lentilles $l$ et $L$ étant respectivement de l'ordre de $10^{-7}$ et $1 \mathrm{H} \Delta I / I$ est de l'ordre de $10^{-7}$. Avec des interrupteurs plus inductifs $l$ peut rapidement valoir $10^{-5} \mathrm{H}$ et la variation $\Delta I / I \simeq 10^{-5}$ peut alors être du même ordre de grandeur que celle que l'on veut commander.

Cette première méthode nécessite en résumé une alimentation très stable, des temporisations importantes avant piégeage d'un courant et une prise en compte de la variation systématique de ce courant après piégeage : elle n'est donc pas très intéressante et présente des risques d'erreur assez importants. Aussi est-il préférable d'opérer différemment sans réouvrir la boucle supraconductrice en utilisant la seconde méthode suivante.

3. Commande indirecte avec un transformateur à courant continu. - Dans cette seconde méthode le circuit supraconducteur reste fermé et on y induit des courants permanents à l'aide d'un transformateur à courant continu dont le secondaire supraconducteur est en série avec l'enroulement de la lentille dans la boucle supraconductrice fermée (Fig. 3).

Dans notre lentille-objectif en particulier, l'interrupteur thermique de supraconduction à une faible inertie et on piège souvent au passage le courant qui correspond à la mise au point : l'intensité piégée est alors notablement différente de l'intensité commandééc et pour effectuer les réglages fins auţür de cettê mise au point, nous opérons selon cette méthode, à l'aide d'un transformateur à courant continu. 


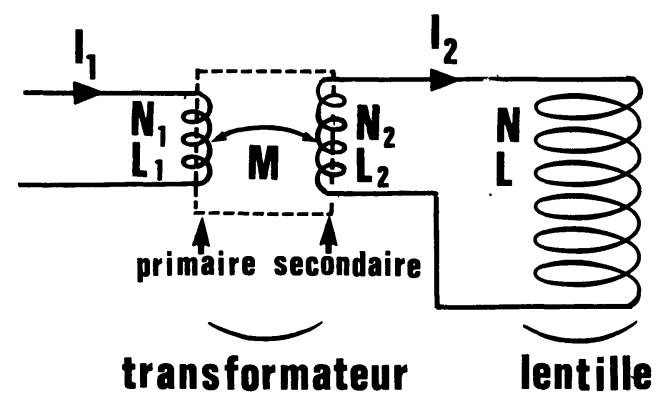

FIG. 3. - Transformateur pour induire des variations de courants dans une lentille en boucle fermée.

Equations du transformateur : considérons les enroulements primaire et secondaire du transformateur et l'enroulement de la lentille et notons respectivement $N_{1}, N_{2}$ et $N$ leurs nombres de tours, $L_{1}, L_{2}$ et $L$ leurs coefficients d'inductance, $I_{1}, I_{2}$ et $I$ les intensités qui les parcourent, $\Phi_{1}, \Phi_{2}$ et $\Phi$ les flux magnétiques qui les traversent. Notons par ailleurs $M$ et $k$ les coefficients d'induction mutuelle et de couplage entre les enroulements du transformateu $\mathrm{r}\left(M=k \sqrt{L_{1} L_{2}}\right)$.

Nous pouvons exprimer les flux par les relations :

$$
\begin{aligned}
& \Phi_{1}=N_{1} \varphi_{1}=M I_{2}+L_{1} I_{1} \\
& \Phi_{2}=N_{2} \varphi_{2}=L_{2} I_{2}+M I_{1} \\
& \Phi=N \varphi=L I_{2} .
\end{aligned}
$$

Le secondaire du transformateur et la lentille constituant un circuit entièrement supraconducteur fermé, le flux total à travers ce circuit $\Phi_{\mathrm{T}}=\Phi_{2}+\Phi$ est donc constant, ce qu'on traduit par :

$$
\Delta \Phi_{\mathrm{T}}=\left(L_{2}+L\right) \Delta I_{2}+M \Delta I_{1}=0 .
$$

L'excitation initiale du primaire étant nulle, on a $\Delta I_{1}=I_{1}$ et on peut écrire l'équation précédente sous la forme :

$$
\frac{\Delta I_{2}}{I_{2}}=\frac{k \sqrt{L_{1} L_{2}}}{L_{2}+L} \cdot \frac{I_{1}}{I_{2}} .
$$

Rappelons que pratiquement on calculera le transformateur pour avoir une gamme de défocalisations de quelques microns ce qui correspond à une variation relative du courant de la lentille de l'ordre du millième $\left(\Delta I_{2} / I_{2}=10^{-3}\right)$.

Nous allons maintenant considérer successivement les transformateurs sans circuit magnétique et les transformateurs avec circuit magnétique avec et sans entrefer.

4. Transformateur sans circuit magnétique. - Dans cette première solution les enroulements du transformateur sont simplement couplés par voisinage. Si on réalise un couplage serré on peut avoir un coefficient $k$ voisin de l'unité. D'autre part l'inductance $L$ de la lentille qui a une carcasse métallique et un grand nombre de tours est grande devant $L_{1}$ et $L_{2}$. Pour simplifier encore et préciser les ordres de grandeur pour ce trans- formateur on peut assimiler ses bobinages à deux solenoïdes identiques de section $S$, de longueur $D$, comportant chacun une couche de $N$ spires en fil de diamètre $d$ et ayant des coefficients d'inductance $L_{1}=L_{2} \simeq \mu_{0} N^{2} S / D=\mu_{0} N S / d$. Dans ces conditions l'équation (3) peut s'écrire :

$$
\frac{\Delta I_{2}}{I_{2}} \simeq \frac{\sqrt{L_{1} L_{2}}}{L} \cdot \frac{I_{1}}{I_{2}} \simeq \frac{\mu_{0} N S}{d \Phi} \cdot I_{1} .
$$

Soit par exemple $L=0,4 \mathrm{H}$ !'inductance de la lentille parcourue par une intensité $I_{2}=10 \mathrm{~A}\left(\Phi=L I_{2}=4 \mathrm{~Wb}\right)$. $\mathrm{Si}$ on réalise le primaire en fil de cuivre de diamètre $d=0,3 \mathrm{~mm}$, le courant qu'on y envoie est environ $I_{1}=300 \mathrm{~mA}$. On a alors $\Delta I_{2} / I_{2}=3 \times 10^{-4}(N S)$ ce qui nécessite une surface $N S$ d'environ $1 \mathrm{~m}^{2}$ si on souhaite une défocalisation de l'ordre de $1 \mu \mathrm{m}$ : on prendra par exemple 100 spires de $12 \mathrm{~cm}$ de diamètre.

J. L. Balladore [6] a réalisé un transformateur de ce type pour la lentille-objectif supraconductrice d'un microscope $300 \mathrm{kV}$ : ses bobinages réalisés sur un cylindre en cuivre de diamètre $15 \mathrm{~cm}$ et de hauteur $5 \mathrm{~cm}$ sont caractérisés par $N_{1}=120, N_{2}=100, L_{1}=1 \mathrm{mH}$ et $L_{2}=3 \mathrm{mH}$. Avec $I_{1}=100 \mathrm{~mA}$ dans le primaire il obtient une variation relative d'intensité dans la lentille $\Delta I_{2} / I_{2} \simeq 1,5 \times 10^{-4}$.

Ce type de transformateur présente deux inconvénients principaux :

- tout d'abord il est encombrant et son placement dans le cryostat est une première difficulté.

- d'autre part, il crée un champ magnétique important et on doit s'arranger pour que ce champ n'ait aucune action parasite sur le faisceau d'électrons. Il n'est pas question de prévoir un blindage car on adopte alors la solution d'un transformateur avec éléments magnétiques. On doit donc éloigner le transformateur de la lentille et pour bénéficier de l'effet d'écran de diverses structures ferromagnétiques du microscope on est amené à le placer à l'extérieur ce qui introduit une complication importante dans la conception du cryostat. Pour éviter ces inconvénients on est amené à concevoir le transformateur différemment en adoptant les solutions suivantes avec circuit magnétique.

5. Transformateur avec circuit magnétique sans entrefer. - Dans cette solution les enroulements du transformateur sont disposés sur un circuit magnétique fermé. Dans la pratique pour réduire le champ magnétique extérieur on adoptera un circuit magnétique de révolution autour de deux enroulements coaxiaux (Fig. 4).

Le circuit magnétique en augmentant de façon importante les coefficients d'inductance $L_{1}$ et $L_{2}$ des enroulements nous permet d'obtenir un rapport de transformation en courant $T$ plus important :

$$
T=\frac{k \sqrt{L_{1} L_{2}}}{L_{2}+L} .
$$




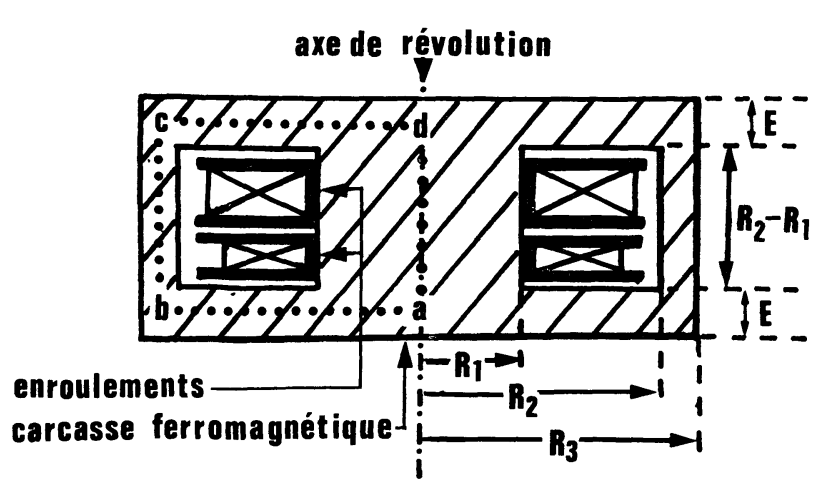

FIG. 4. - Section méridienne d'un transformateur avec carcasse ferromagnétique sans entrefer.

On peut écrire les coefficients d'inductance sous la forme $L_{1}=a_{1} N_{1}^{2}$ et $L_{2}=a_{2} N_{2}^{2}$ : les enroulements étant du même type les coefficients $a_{1}$ et $a_{2}$ ne sont pas très différents et pour simplifier on prendra $a_{1}=a_{2}=a$. On a d'autre part le coefficient de couplage $k$ qui est voisin de l'unité et $L_{2}$ qui est toujours très petit devant $L$ même pour un transformateur avec circuit magnétique. On sait enfin que le coefficient $a$ est proportionnel à la section $S$ des enroulements et à la perméabilité $\mu$ du milieu. Dans ces conditions le rapport de transformation a pour expression :

$$
T \simeq \frac{a N_{1} N_{2}}{L}=\frac{b \mu S N_{1} N_{2}}{L}
$$

On voit que pour une même valeur de $T$ un accroissement de la perméabilité $\mu$ permet une réduction de la section ou des nombres de tours des enroulements donc une réduction des dimensions du transformateur. Comme nous allons le voir cette solution avec circuit magnétique va en effet permettre une miniaturisation du transformateur ce qui sera intéressant tant du point de vue de l'encombrement que du point de vue de la masse à refroidir qui restera faible malgré l'emploi du fer.

L'induction magnétique dans la carcasse du transformateur suit la courbe de variation présentée sur la figure 5 qui donne l'induction magnétique $B$ dans du fer doux en fonction de l'excitation magnétique $H$ et sur laquelle on peut schématiquement distinguer deux zones. Dans la première $B$ atteint assez rapidement 1,5 T pour $H=800 \mathrm{~A} / \mathrm{m}$ avec une pente de l'ordre de $10^{-3}$ MKSA. Dans la seconde $B$ croît beaucoup plus lentement selon la loi approchée $B \simeq 0,85 \mathrm{H}^{0,085}$ : la pente est comprise entre $10^{-4}$ et $10^{-6} \mathrm{MKSA}$ et il faut une excitation $H \simeq 24000 \mathrm{~A} / \mathrm{m}$ pour atteindre $B=2 \mathrm{~T}$.

Quand le primaire du transformateur n'est pas alimenté le circuit magnétique est cependant déjà excité par le secondaire parcouru par l'intensité $I_{2}$, piégée dans la lentille. Cette excitation initiale $N_{2} I_{2}$ détermine le point de fonctionnement $M_{0}\left(H_{0}, B_{0}\right)$ du transformateur. A priori il semble intéressant d'avoir pour le transformateur une dynamique importante et donc une

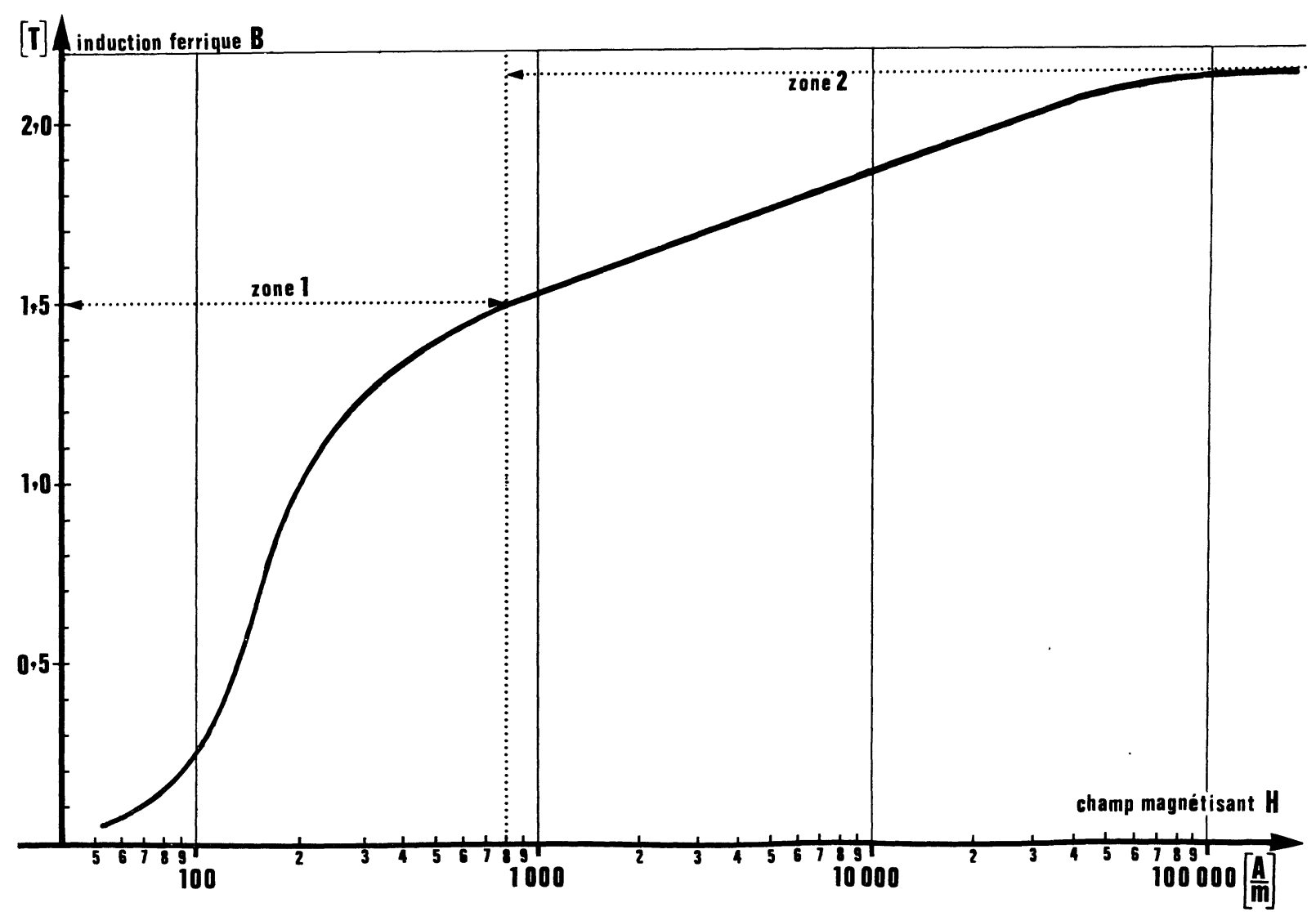

FIG. 5. - Courbe d'aimantation du fer pur ARMCO. 
induction initiale $B_{0}$ pas trop grande : comme nous le verrons par la suite nous obtiendrons dans ce cas des solutions avec un faible nombre de tours $N_{2}$ au secondaire. Nous trouverons cependant également des solutions de très faible encombrement avec $N_{2}$ et $B_{0}$ importants, les dynamiques étant alors restreintes à quelques 0,1 T. Nous allons établir ci-dessous des relations approchées simples permettant de déterminer les ordres de grandeur des variables électriques et géométriques caractérisant ces diverses solutions de transformateur.

5.1 Relations GÉOMÉTRIQUES. - Pour simplifier le problème nous considérerons un transformateur dans lequel l'espace torique disponible pour les enroulements est à section carrée. La figure 4 présente la section méridienne d'un tel transformateur dont nous caractérisons la géométrie par les quatre longueurs $R_{1}$, $R_{2}, R_{3}$ et $E$.

Nous devons tout d'abord choisir ces variables de façon à ce que le circuit magnétique offre à peu près une même section aux lignes de champ schématisées sur la figure 4 par le parcours moyen $(a b c d a)$ : l'excitation magnétique dont l'ordre de grandeur est le même sur tout ce parcours peut alors être évaluée de façon simple. La conservation de cette section au niveau des points $(a)$ et $(b)$ conduit à :

$$
\begin{aligned}
E & =\frac{R_{1}}{2} \\
R_{3} & =\sqrt{\left(R_{1}+R_{2}\right) R_{2}} .
\end{aligned}
$$

Nous devons ensuite définir les conditions pour que l'on puisse loger les bobinages d'excitation à l'intérieur de la carcasse. Soient $S_{1}=N_{1} I_{1} / J_{1}$ et $S_{2}=N_{2} I_{2} / J_{2}$ les sections des enroulements primaire et secondaire du transformateur ( $N I$ est le nombre d'ampère tours d'excitation et $J$ la densité de courant). Ces enroulements sont réalisés sur deux mandrins dont on doit prendre en compte l'encombrement : si leur épaisseur est $X$ leur section d'encombrement est approximativement $S_{3} \simeq 5 X\left(R_{2}-R_{1}\right)$. Les bobinages en question doivent être logés dans l'espace disponible de section $S_{4}=\left(R_{2}-R_{1}\right)^{2}$, la condition de placement étant $S_{4}=S_{1}+S_{2}+S_{3}$ soit :

$\left(R_{2}-R_{1}\right)\left(R_{2}-R_{1}-5 X\right)-\frac{N_{1} I_{1}}{J_{1}}-\frac{N_{2} I_{2}}{J_{2}}=0$.

Nous pouvons finalement expliciter la longueur $D \mathrm{du}$ parcours $(a b c d a)$ soit $D=\sqrt{\left(R_{1}+R_{2}\right) R_{2}}+3 R_{2}-R_{1}$. Nous utiliserons cette relation pour déterminer $R_{2}$ connaissant $D$ et $R_{1}$ et nous la considérerons donc sous la forme d'une équation du second degré en $R_{2}$ (une seule solution convenant) :

$$
8 R_{2}^{2}-\left(6 D+7 R_{1}\right) R_{2}+\left(D+R_{1}\right)^{2}=0 .
$$

Nous disposons à ce stade de quatre équations (9 à 12) pour les sept inconnues $R_{1}, R_{2}, R_{3}, E, D, N_{1} I_{1}$ et $N_{2}$, les quantités $J_{1}, J_{2}, I_{2}$ et $X$ étant données.
5.2 Relations ÉLECTRIQUES. - Nous raisonnerons de façon simple comme si les dimensions transversales du circuit magnétique étaient petites par rapport à sa longueur $D$ et nous appliquerons alors le théorème d'Ampère au parcours $(a b c d a)$ pour déterminer l'excitation magnétique $H$ supposée uniforme sur toute la section du circuit : si on s'écarte de ces conditions on obtiendra cependant de bons ordres de grandeur pour la réalisation du transformateur.

Soit $M_{0}\left(H_{0}, B_{0}\right)$ le point de fonctionnement du transformateur. Sa position est déterminée par l'excitation initiale $\mathrm{H}_{0}$ due aux ampère-tours $\mathrm{N}_{2} \mathrm{I}_{2}$ du bobinage secondaire :

$$
\mathrm{N}_{2} \mathrm{I}_{2} \simeq \mathrm{H}_{0} \mathrm{D} \text {. }
$$

Soit $M_{\mathrm{M}}\left(H_{\mathrm{M}}, B_{\mathrm{M}}\right)$ le point extrême de la dynamique du transformateur. Ce point est atteint avec un nombre d'ampère-tours au primaire $N_{1} I_{1}$ donné par

$$
N_{1} I_{1} \simeq\left(H_{\mathrm{M}}-H_{0}\right) D \text {. }
$$

Soit $\Delta \Phi$ la variation de flux totale qu'on veut créer dans le circuit de la lentille supraconductrice :

$$
\Delta \Phi=N_{2} \Pi R_{1}^{2}\left(B_{\mathrm{M}}-B_{0}\right) .
$$

La variation de flux à créer $\Delta \Phi$ étant une donnée et l'excitation $H$ étant numériquement connue en fonction de l'induction $B$ nous n'avons donc introduit que deux inconnues supplémentaires $B_{0}$ et $B_{\mathrm{M}}$ ce qui porte à neuf leur nombre total. Ne disposant que de sept équations ( 9 à 15) nous devrons donc prendre deux de ces inconnues comme paramètres dans la résolution $\mathrm{du}$ problème. Nous choisirons en premier $B_{M}$ dont l'ordre de grandeur est connu et qui sera par exemple égal à 1,5 ou $2 \mathrm{~T}$ selon qu'on fait ou non travailler le transformateur dans la zone 2 de la courbe $B(H)$. Nous prendrons en second le nombre de tours $N_{2}$ du secondaire supraconducteur et nous choisirons parmi les solutions obtenues celles qui ont un faible encombrement et un nombre de tours $N_{2}$ pas trop important.

Nous avons regroupé les équations du problème dans un petit programme de calcul sur ordinateur qui nous permet de déterminer les caractéristiques optimum d'un transformateur de performance donnée, en fonction de l'un ou des deux paramètres du problème.

5.3 CAlcul D'Un TRANSFormateur. - La lentille supraconductrice de notre microscope $400 \mathrm{kV}$ a une distance focale $f_{0} \simeq 2 \mathrm{~mm}$, un coefficient d'inductance $L \simeq 0,5 \mathrm{H}$ et elle est parcourue par un courant d'intensité $I_{2} \simeq 10 \mathrm{~A}$ : le flux dans le circuit de cette lentille est donc $\Phi=L I_{2} \simeq 5 \mathrm{~Wb}$.

On veut réaliser un transformateur permettant de commander dans cette lentille des défocalisations allant jusqu'à $1 \mu \mathrm{m}$ : on doit pour cela déterminer une variation relative de flux $\Delta \Phi / \Phi \simeq 4 \times 10^{-4}$ soit dans le cas présent une variation absolue $\Delta \Phi \simeq 2 \times 10^{-3} \mathrm{~Wb}$.

On prendra par ailleurs $J_{2}=10^{8} \mathrm{Am}^{-2}$ dans le secondaire, $J_{1}=10^{7} \mathrm{Am}^{-2}$ dans le primaire en cuivre et $X=0,5 \mathrm{~mm}$ pour l'épaisseur des mandrins. 
L'ensemble des solutions possibles est présenté par le réseau de courbes de la figure 6 donnant le volume du transformateur en fonction des paramètres $B_{\mathrm{M}}$ et $N_{2}$. Les solutions d'encombrement minimum et l'induction maximum correspondante sont données en fonction de $N_{2}$ sur les courbes de la figure 7 .

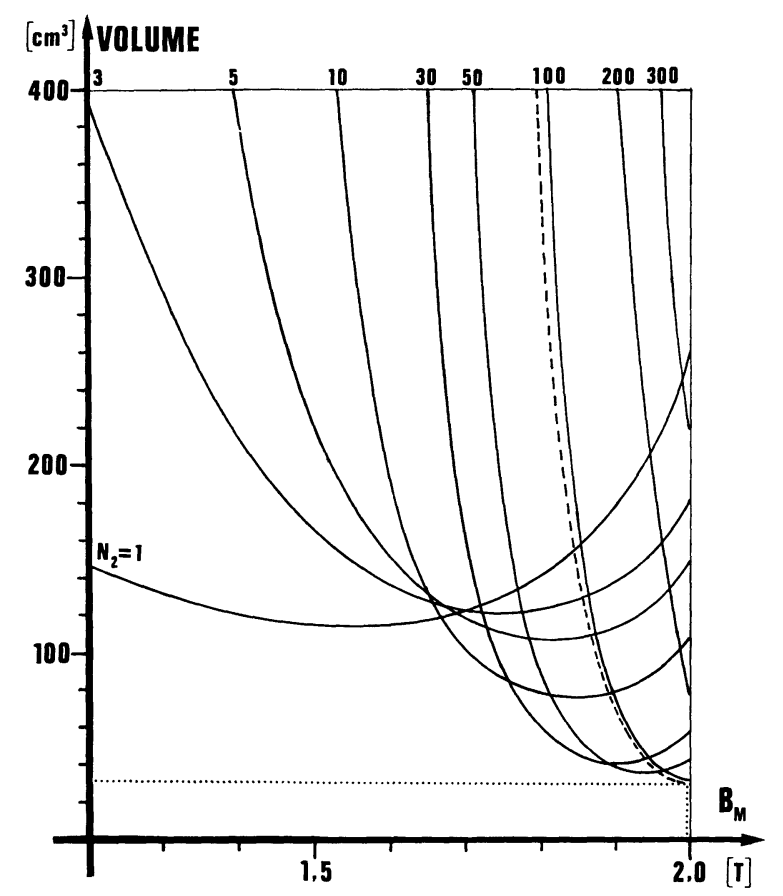

FIG. 6. - Encombrement des modèles sans entrefer calculés pour une variation du flux $\Delta \Phi=2 \times 10^{-3} \mathrm{~Wb}$.

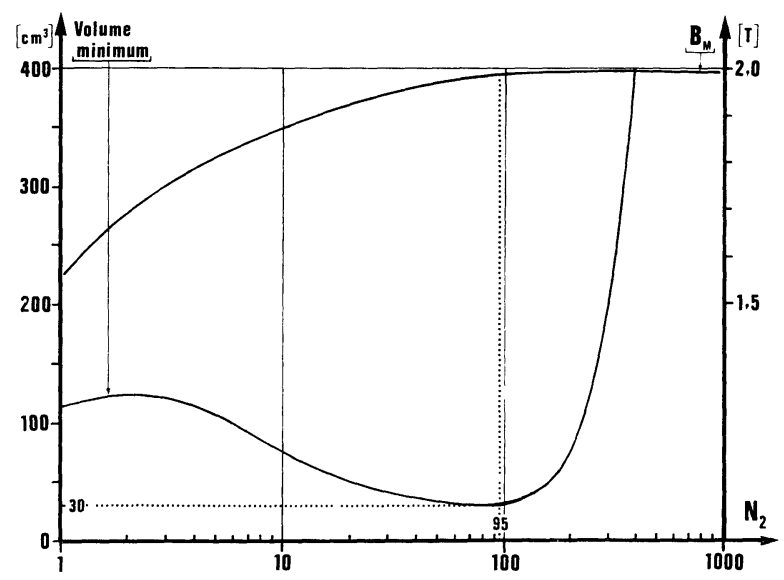

FIG. 7. - Encombrement minimum des modèles sans entrefer en fonction du nombre de tours $N_{2}$ au secondaire.

Le transformateur le plus petit est obtenu pour $N_{2}=95$ et $B_{\mathrm{M}}=2 \mathrm{~T}$. Sa dynamique va de 1,8 à $2 \mathrm{~T}$ pour l'induction $B(7000$ à $24000 \mathrm{~A} / \mathrm{m}$ pour l'excitation $H$ ) avec un nombre d'ampère-tours $N_{2} I_{2}=950 \mathrm{~A}$.t au secondaire et $N_{1} I_{1}= \pm 700$ A.t au primaire. Son volume est de $30 \mathrm{~cm}^{3}$ et ses dimensions sont présentées comparativement sur la figure 13.
6. Transformateur avec circuit magnétique avec entrefer. - On peut introduire un entrefer dans le circuit magnétique du transformateur pour que la saturation du fer intervienne moins rapidement avec l'excitation. Cela doit permettre d'une part une réduction d'encombrement pour le transformateur et cela détermine d'autre part la linéarisation de sa réponse si l'entrefer est bien calculé et que la perméabilité du fer reste importante.

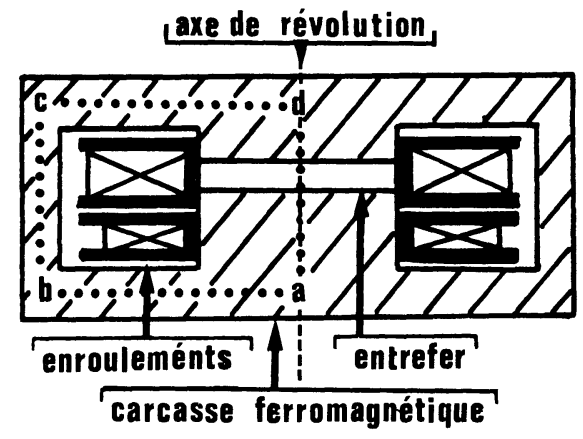

FIG. 8. - Section méridienne d'un transformateur avec carcasse ferromagnétique avec entrefer.

6. 1 ÉQUATIONS DU PROBLÈME. - Considérons sur la figure 8 un circuit magnétique avec un entrefer sur le noyau central. Appliquons le théorème d'Ampère au parcours $(a b c d a)$ dans les mêmes conditions que précédemment en notant $\mu_{\mathrm{r}}$ la perméabilité relative du fer, $D$ et $d$ les longueurs des parcours dans le fer et dans l'air :

$$
B\left(\frac{D}{\mu_{\mathrm{r}}}+d\right)=\mu_{0} N I .
$$

La réponse du transformateur est approximativement linéaire si le terme $D / \mu_{\mathrm{r}}$ est négligeable devant le terme $d$ et on a alors :

$$
B \simeq \frac{\mu_{0} N I}{d}
$$

Si on tolère un écart de linéarité de $10 \%$ la condition s'écrit :

$$
D<\frac{\mu_{\mathrm{r}}}{10} d
$$

Nous présentons sur la figure 9 la variation de la perméabilité relative du fer $\mu_{\mathrm{r}}$ en fonction de l'induction $B$. Pour $B_{\mathrm{M}} \simeq 2 \mathrm{~T}$ on a $\mu_{\mathrm{r}} \simeq 100$ et la condition s'écrit $D<10 d$ : cela nous conduit à prendre un entrefer important pour que $D$ soit compatible avec le placement des enroulements. Si par contre on se limite à $B_{\mathrm{M}} \simeq 1,5 \mathrm{~T}$ on a $\mu_{\mathrm{r}}>2000$ et la condition s'écrit $D<200 . d$ : cela autorise des valeurs plus faibles de l'entrefer et donc une meilleure efficacité du transformateur.

L'induction $B$ dans le circuit du transformateur varie linéairement en fonction du nombre d'ampère-tours d'excitation $A$ (Fig. 10). Sa valeur $B_{0}$ au point de fonc- 


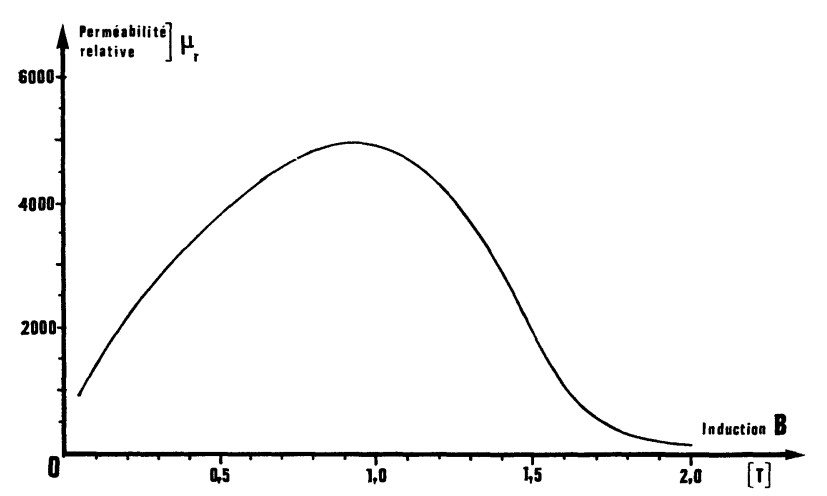

FIG. 9. - Perméabilité magnétique relative du fer pur ARMCO en fonction de l'induction ferrique.

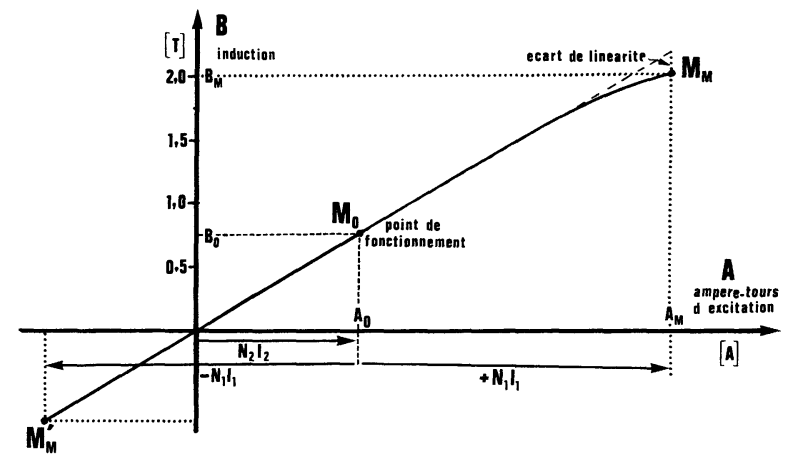

Fig. 10. - Courbe de réponse linéaire d'un transformateur avec carcasse ferromagnétique avec entrefer.

tionnement du transformateur est fixée par l'excitation $A_{0}=N_{2} I_{2}$ due au bobinage secondaire. Sa valeur maximum $B_{\mathrm{M}}$ est atteinte pour une excitation totale $A_{\mathrm{M}}$. La variation totale du flux dans l'enroulement secondaire a donc pour expression :

$\Delta \Phi=N_{2}\left(B_{\mathrm{M}}-B_{0}\right) S=\frac{\mu_{0} S}{d}\left(A_{\mathrm{M}} N_{2}-I_{2} N_{2}^{2}\right)$.

Cette variation de flux est maximum en fonction de $\mathrm{N}_{2}$ quand $A_{\mathrm{M}}=2 \mathrm{~N}_{2} I_{2}$, c'est-à-dire pour des nombres d'ampère-tours d'excitation identiques au primaire et au secondaire du transformateur : $N_{1} I_{1}=N_{2} I_{2}$.

Comme dans le cas précédent nous avons fait un programme de calcul pour optimiser le transformateur. Les grandeurs $I_{2}, J_{1}, J_{2}, \Delta \Phi$ et $X$ étant données, $N_{2}$ et $B_{\mathrm{M}}$ sont encore pris comme paramètres et on utilise dans le cas présent les équations suivantes :

$$
\begin{aligned}
N_{1} I_{1}= & N_{2} I_{2} \\
R_{1}= & \sqrt{\frac{2 \Delta \Phi}{\Pi N_{2} B_{\mathrm{M}}}} \\
R_{2}^{2}- & \left(2 R_{1}+5 X\right) R_{2}+ \\
& +\left(R_{1}^{2}+5 X R_{1}-\frac{N_{1} I_{1}}{J_{1}}-\frac{N_{2} I_{2}}{J_{2}}\right)=0 \\
R_{3}= & \sqrt{\left(R_{1}+R_{2}\right) R_{2}} \\
d= & \frac{2 \mu_{0} N_{2} I_{2}}{B_{\mathrm{M}}} .
\end{aligned}
$$

Les solutions obtenues conviennent uniquement si on a :

$$
D=R_{3}+3 R_{2}-R_{1}-d<\frac{\mu_{\mathrm{r}}}{10} d
$$

6.2 CAlCul D'Un TRANSFormateur. - Les calculs sont faits avec les mêmes données numériques que pour le modèle sans entrefer. L'ensemble des solutions est présenté par les courbes de la figure 11 qui donnent le volume du transformateur en fonction des paramètres $N_{2}$ et $B_{\mathrm{M}}$. Ces courbes son limitées à gauche (points $a$, $b, c, d, e, f, g, \ldots)$ à cause de la condition d'écart à la linéarité qu'on s'est proposée (inférieure à $10 \%$ ). Audelà de $B_{\mathrm{M}}=1,6 \mathrm{~T}$ les portions de courbes sont très voisines et à cause des limitations elles ne présentent plus de vrais minimum. La figure 12 présente les courbes donnant l'encombrement minimum et l'induction $B_{\mathrm{M}}$ correspondante en fonction de $N_{2}$.

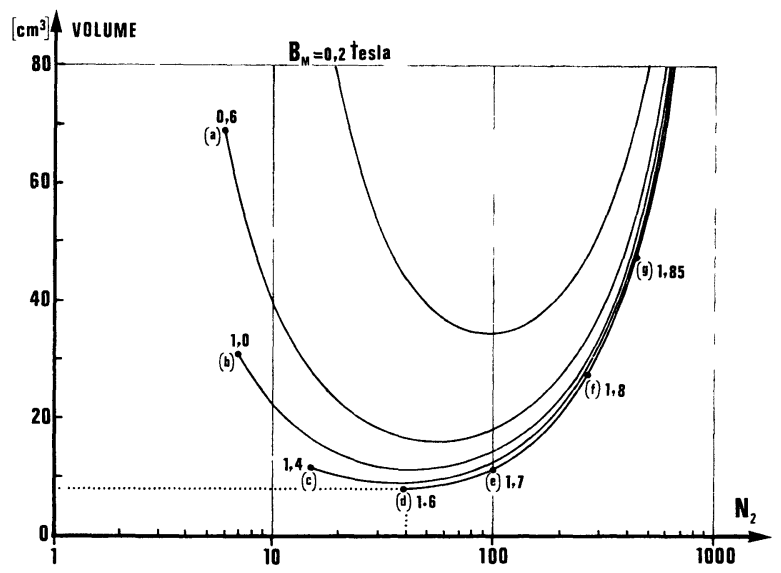

Fig. 11. - Encombrement des modèles avec entrefer calculés pour une variation de flux $\Delta \Phi=2 \times 10^{-3} \mathrm{~Wb}$.

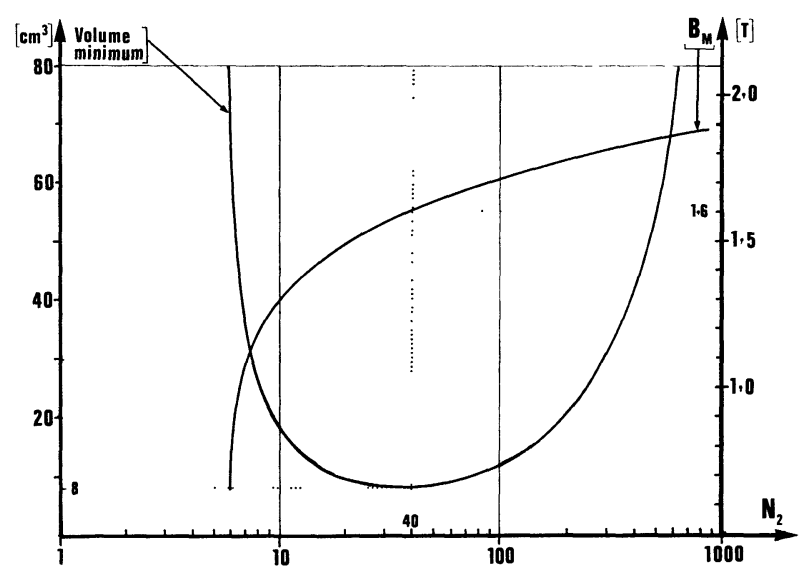

FIG. 12. - Encombrement minimum des modèles avec entrefer en fonction du nombre de tours $N_{2}$ au secondaire.

Le transformateur le plus petit a un volume de $8 \mathrm{~cm}^{3}$ : il intervient pour $N_{2}=40, B_{\mathrm{M}}=1,6 \mathrm{~T}$ et $d=0,6 \mathrm{~mm}$. Sa dynamique va de $B=0$ à $B=1,6 \mathrm{~T}$ 
avec $N_{2} I_{2}=400$ A.t au secondaire et $N_{1} I_{1}= \pm 400$ A.t au primaire. Ses dimensions sont présentées sur la figure 13.

7. Etude expérimentale des solutions calculées. Les transformateurs avec carcasses ferromagnétiques sont les plus intéressants car ils sont très peu encombrants. Sur la figure 13 nous présentons les sections méridiennes des deux transformateurs que nous avons calculés précédemment : le modèle avec entrefer, beaucoup plus petit que le modèle sans entrefer est le plus intéressant.

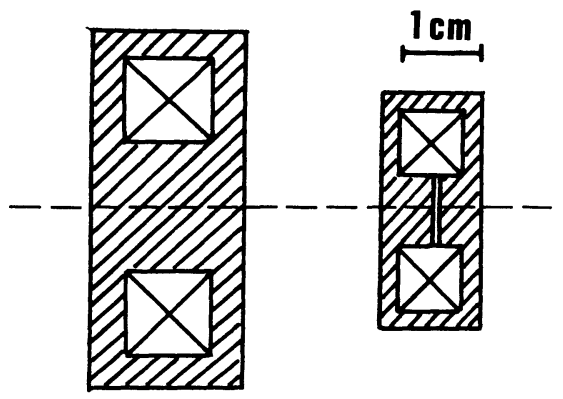

(a)

(b)

Fig. 13. - Sections à l'échelle des transformateurs d'encombrement minimum sans entrefer $(a)$ et avec entrefer $(b)$ pour une variation de flux $\Delta \Phi=2 \times 10^{-3} \mathrm{~Wb}$.

Pour évaluer l'exactitude de nos calculs nous avons réalisé ces deux transformateurs qu'on peut voir sur la figure 14 et nous avons déterminé expérimentalement leurs courbes de réponse donnant leurs flux $\Phi$ en fonction de leurs excitations.

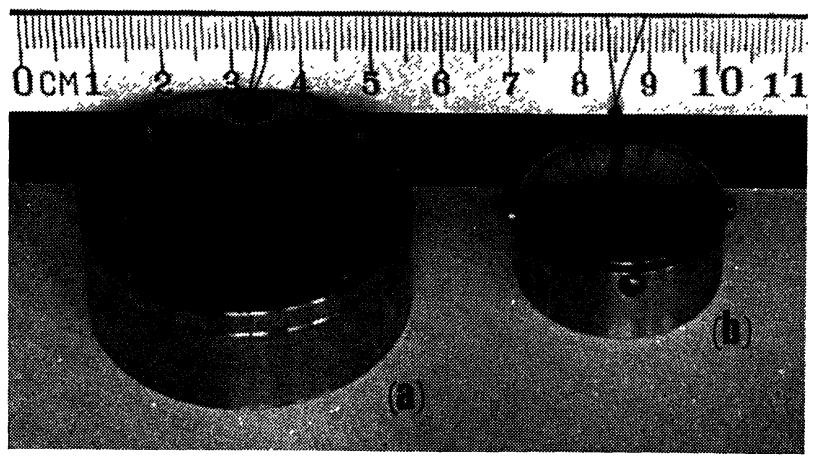

FIG. 14. - Vue des transformateurs optimisés étudiés expérimentalement (modèles sans entrefer $(a)$ et avec entrefer $(b)$ ).

La courbe de réponse du modèle sans entrefer est donnée sur la figure 15 . Son point de fonctionnement correspond à une excitation secondaire $N_{2} I_{2}=950 \mathrm{~A} . \mathrm{t}$ et à un flux $\Phi=40 \mathrm{mWb}$. De part et d'autre de ce point, pour une excitation primaire $N_{1} I_{1}= \pm 725 \mathrm{~A} . \mathrm{t}$ on a les variations de flux $\Delta \Phi=+3 \mathrm{mWb}$ et - $10 \mathrm{mWb}$ : ces performances sont meilleures que prévues puisque le transformateur a été calculé pour une variation de flux $\Delta \Phi=+2 \mathrm{mWb}$ vers les excitations croissantes.

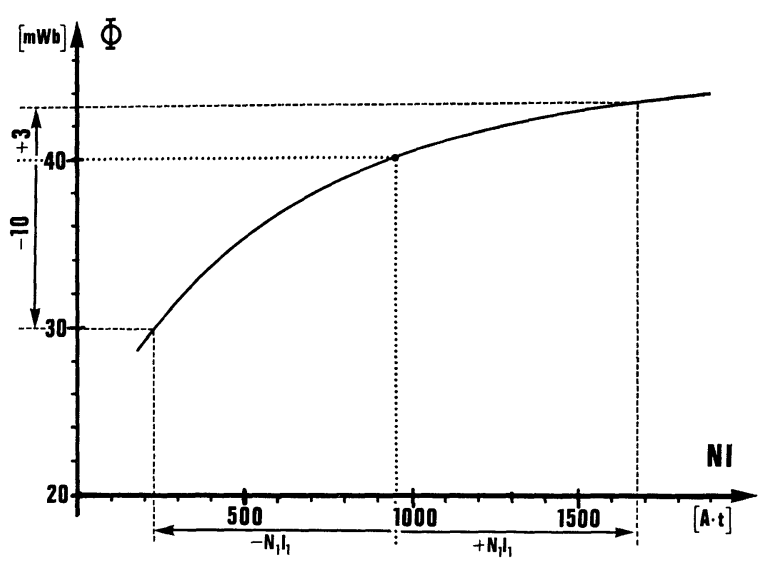

FIG. 15. - Courbe de réponse expérimentale du modèle optimisé sans entrefer.

La courbe de réponse du modèle avec entrefer est donnée sur la figure 16. Son point de fonctionnement est défini par $N_{2} I_{2}=400$ A.t et $\Phi=3 \mathrm{mWb}$. Pour une excitation au primaire $N_{1} I_{1}= \pm 400 \mathrm{~A} . \mathrm{t}$ on a les variations de flux $\Delta \Phi=+2,6 \mathrm{mWb}$ et $-3 \mathrm{mWb}$ qui sont donc également supérieures aux prévisions théoriques.

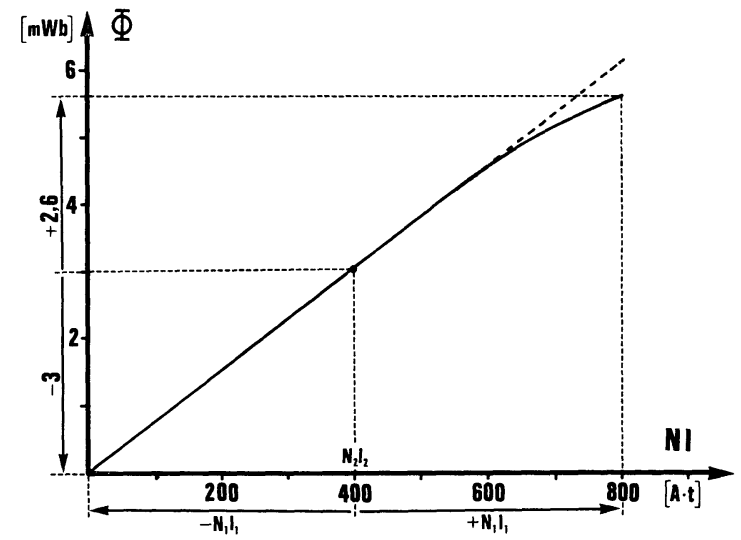

FIG. 16. - Courbe de réponse expérimentale du modèle optimisé avec entrefer.

Actuellement la lentille-objectif de notre microscope supraconducteur $400 \mathrm{kV}$ est équipée du transformateur avec entrefer calculé ci-dessus : nous présentons sur la figure 17 les images sous-focalisée, au point et surfocalisée d'un trou d'une membrane, obtenues en utilisant ce transformateur.

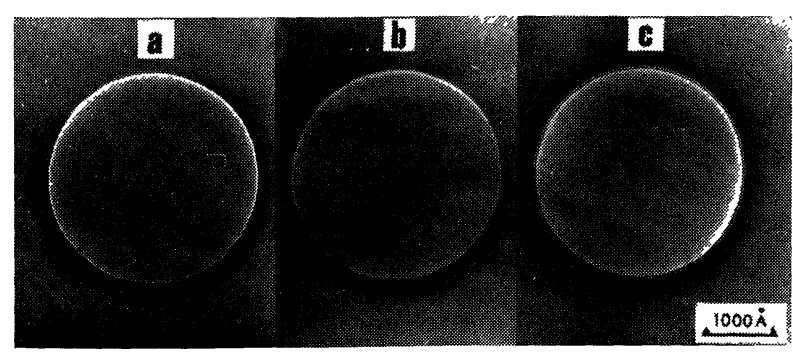

FIG. 17. - Microphotographies d'un trou illustrant l'action du transformateur de notre microscope. Les défocalisations des vues (a), (b), (c) sont respectivement $\Delta z=-1,5 ; 0$ et $+1,5 \mu \mathrm{m}$. 
8. Instabilité en courant induite dans la lentille par le transformateur. - Nous noterons avec $« \Delta »$ les variations permanentes des courants et avec $« \delta »$ leurs instabilités. Soit $I$ le courant parfaitement stable piégé dans la lentille $(\delta I=0)$. Quand on envoie un courant $I_{1}$ dans le primaire du transformateur on induit une variation de courant $\Delta I$ dans la lentille parcourue alors $\operatorname{par} I_{2}=I+\Delta I$.

Soit $\delta I_{1}$ l'instabilité du courant dans le primaire et $\delta I_{2}=\delta(I+\Delta I)=\delta(\Delta I)$ l'instabilité du courant dans la lentille. En valeurs relatives on a :

$$
\frac{\delta I_{2}}{I_{2}}=\frac{\delta(\Delta I)}{I+\Delta I} \simeq \frac{\delta(\Delta I)}{I}=\frac{\delta(\Delta I)}{\Delta I} \cdot \frac{\Delta I}{I}
$$

La variation $\Delta I$ étant donnée par la relation $\Delta I=T . I_{1}$ où $T$ est le rapport de transformation en courant, on a $\delta(\Delta I) / \Delta I=\delta I_{1} / I_{1}$, soit finalement :

$$
\frac{\delta I_{2}}{I_{2}}=\frac{\delta I_{1}}{I_{1}} \cdot \frac{\Delta I}{I} .
$$

L'instabilité relative du courant dans la lentille est donc égale à celle du courant dans le primaire multipliée par le taux de courant induit $\Delta I / I$. Dans la pra- tique pour une défocalisation d'un micron ce taux est de l'ordre de $5 \times 10^{-4}$ et l'instabilité de la lentille peut toujours de ce fait être rendue parfaitement négligeable.

Pour avoir une stabilité parfaite au primaire on a d'ailleurs toujours la possibilité de réaliser l'enroulement primaire avec du fil supraconducteur et de le faire fonctionner en boucle fermée. Indépendamment de cette stabilité, cette solution est intéressante parce qu'elle permet une petite réduction d'encombrement et surtout parce que la puissance électrique et l'ébullition d'hélium qu'elle engendre sont alors nulles dans le transformateur.

9. Conclusion. - Le réglage fin de l'excitation d'une lentille supraconductrice en boucle fermée nécessite l'emploi d'un transformateur à courant continu. Nous avons établi quelques relations simples permettant d'optimiser ces transformateurs et nous avons montré que le modèle à carcasse ferromagnétique avec entrefer était le plus intéressant. Nous avons comme exemple déterminé les modèles optimisés avec et sans entrefer pour la lentille-objectif de notre microscope supraconducteur $400 \mathrm{kV}$ : leur étude expérimentale a montré la validité de nos calculs dont les prévisions sont en bon accord avec les performances mesurées.

\section{Bibliographie}

[1] Laberrigue, A., Severin, C., J. Microsc. 6 (1967) 123.

[2] Genotel, D., Severin, C., Laberrigue, A., J. Microsc. 7 (1967) 933.

[3] Genotel, D., Laberrigue, A., Payen, F., Severin, C., Electron Microscopy, Rome, 1968, 187.
[4] Severin, C., Genotel, D., Girard, M., Laberrigue, A. Revue Phys. Appl. 6 (1971) 459.

[5] SePtiER, A., Focusing of Charged Particules (Academic Press) 1967, Vol. 1.

[6] Balladore, J. L., Thèse, Toulouse, 1972. 